\title{
Potato Root Eelworm, D-D, and Soil Sterilization. I. Methods and Criteria.
}

By B. G. Peters, M.Sc., Ph.D.

(Nematology Department, Rothamsted Experimental Station, Harpenden).

In the interpretation of field experiments on the use of D-D for controlling the potato root eelworm. Heterodera rostochiensis, various difficulties have arisen. The chemical seemed far less effective in killing the eelworm than might have been expected from results published in the U.S.A., yet on the other hand greater increases in the yield of potatoes were produced than could reasonably be explained by the small proportion of eclworms killed. The whole situation is suggestive of a partial sterilization effect, such as was found in the early work at Rothamsted and elsewhere when volatile organic compounds were injected into soil. In Hawaii, Carter and his colleagues have noted a similar "soil amendment" effect from the injection of D-D.

Whilst this effect is an important factor on the credit side, when assessing the results of D-D injections, it is to be sharply differentiated from the purely nematocidal effect. There must be many organic substances which would give comparable soil amendment effects, reflected in increased yields, without killing eelworms at all, and their continued use could only lead to an increase in the eelworm population, possibly to disastrous levels. It follows that yields cannot be used as a criterion of nematocidal efficacy in such a situation, and that some direct estimation of eelworm population is required instead.

It was considered desirable to explore the effects on the host plant of both steam sterilization and D-D injection, both in the presence and in the absence of eelworm infection. It was therefore decided to sct up a pot experiment in factorial form, making use of the three factors : partial steam-stcrilization of soil (symbolized S), infection of soil with Heterodera cysts $(H)$, and injection of soil with D-D (symbolized D). applied in that order. Each factor was applied to half the pots in such a way that all the eight possible combinations were equally represented : $O, H, S, D, H S, H D, S D$, and HSD. Since 40 large pots were available a 5-fold replication of each of the eight treatments was possible.

There is an anomalous feature about this design since, if D-D should kill all or most of the eelworms in any pot where both factors oxcur together (HD and HSD), the $H$ factor would in fact be absent from 
such pots. After due consideration it was decided to proceed nevertheless and to rely on analysis to solve this problem.

Apart from the primary intention of investigating the effects of these three factors on the host plants, the 20 infected pots $(\mathrm{H})$ lent themselves to a secondary enquiry into the effects of factors $S$ and $D$ on the eelworm population. The primary intention requires a selection of suitable criteria for measuring the effects on the plants.

The present paper sets out the methods used in carrying out the experiment and the way in which suitable criteria were selected, leaving it to a second paper to discuss the results obtained. In the event, these results were considered sufficiently interesting to justify keeping the pots of soil and repeating the experiment, without renewing the treatments, in a second year. These second-year results will be discussed in a third paper.

\section{Methods.}

Soil. There was available a heap of good quality top-spit meadow soil, a medium loam, used for general potting purposes. This was found free from $H$. rostochiensis and was passed through a coarse sieve and divided into two equal portions, one of which was sterilized.

Sterilization was carried out in a small portable apparatus of the low-pressure type. Steam was passed through the soil until it reached a temperature of about $85^{\circ} \mathrm{C}$. when it was allowed to cool and spread on a cemented floor to dry. Both this and the unsterilized soil were separately mixed with clean silver sand in the proportion by volume i4: 5.

Pots were $12^{\prime \prime}$ high and $13^{\prime \prime}$ in diameter, and held $15 \mathrm{Kg}$. of soil to within $2^{\prime \prime}$ of the rim. They had been coated with paraffin wax internally for a previous purpose.

Cysts of Heterodera rostochiensis were taken from a clean stock of about $20 \mathrm{gm}$. which had been previously floated out from an experimental soil: 20 lots of $800.0 \mathrm{mg}$. were weighed out for the experiment, and also 5 lots of $100.0 \mathrm{mg}$. for counting. The counts showed that $800 \mathrm{mg}$. would contain $-23,800$ cysts, with a standard error of 440 cysts: Dissection of 50 cysts showed that the mean number of viable eggs and larvae was 122.7 per cyst, or $2.92 \times 10^{\circ}$ per $800 \mathrm{mg}$. $52 \%$ of cysts were empty.

-Infection. Half the sterilized and half the unsterilized pots were taken at random for infection. A potful of soil was spread on a clean cemented floor; the $800 \mathrm{mg}$. of cysts was thoroughly mixed with $50 \mathrm{ml}$. 
of dry silver sand and the mixture evenly sprinkled over the soil, which was mixed by a shovel by coning and quartering and then replaced in the pot.

Injection of D-D into half the pots took place on the same date as infection (2/4/46). $10 \mathrm{ml}$. of D-D was run through a vertical glass tube plunged 6 " deep in the centre of each pot, after which all pots were lightly watered to form a seal. Watering ivas repeated on the second and third days since hot sun was cracking the soil surface. In so far as the area of soil surface can justifiably be compared with field areas, this rate of injection was equivalent to about 122 gallons/acre $(1460 \mathrm{lb}$./acrc). This high rate was selected because of the inconclusive results in field experiments at considerably lower rates.

Potatoes. 40 tubers of fairly even size, averaging $48 \mathrm{gm}$., were selected from a stock of $28 \mathrm{lb}$. Class $A$ "Duke of York" Scotch seed, and lightly scrubbed with a soft brush with water to remove soil particles and cysts (if any). The sieved residue yielded two empty cysts of $H$. rostochiensis. The seed were chitted for 28 days when all eyes but two were rubbed out, and planted 2 " deep in the pots on the 28th day after injection (30/4/46). The pots were stood in four rows of 10 on a cement floor in an open but sheltered courtyard, and were randomized for position without restriction.

\section{Criteria.}

The effects of treatment on the growth of the potato plants were measured by a variety of critcria, namely : (1) Height of highest shoot, to the nearest centimetre, at $29,45,59$, and 78 days after planting. This was measured by means of-a horizontal bar sliding over a vertical rod calibrated in centimetres.

(2) Colour of foliage at 45 days after planting. Scores of $-1,0$ and +1 were allotted respectively to plants showing light, medium, or dark green foliage. It was a matter of some surprise that an ordinary analysis of variance applied to these scores gave significant and sensible results.

(3) After the last height reading $(17 / 7 / 46)$, when the foliage was yellowing and beginning to droop, the haulms were cut off level with the soil and oven-dried in linen bags at $105^{\circ} \mathrm{C}$. to constant weight. The weight in grammes was used as a criterion.

(4) and (5) The number and weight of tubers from each pot were recorded, separating ware-with-seed fron chats. The number of warewith-seed tubers and the total number gave two items for the fourth criterion, and the corresponding two weights gave the fifth. 
(6) In addition to the above plant criteria, this and the following were used for assessing the effects on the eelworm population of the two factors $S$ and $D$, using only the 20 infected pots. Duplicate $200 \mathrm{gm}$. soil samples were taken from the air-dried and thoroughly mixed soil of each pot. These samples were washed in Fenwick's apparatus and the contained cysts floated up, collected and counted.

(7) Duplicate batches of 100 cysts from each soil rample were treated with calcium hypochlorite (Fenwick's method) and each batch made up to $50 \mathrm{ml}$. with water. In each case duplicate $1 \mathrm{ml}$. aliquots were withdrawn by pipette from the stirred volume, and the contained eelworm larvae counted. Thus, the total count for each pot represents in effect "larvae/8 cysts". The eggs failing to hatch in the hypochlorite were not counted.

\section{Analysis.}

The variance between the eight treatment menns was expressed as a ratio to the error variance occurring within batches of 5 replicates, in the usual way, and only where this ratio (F) was significant were the treatments separately assessed. It should perhaps be explained that the data from all 40 pots are used for assessing not only each of the three factors (H, S, D) but also each of the interactions (HS, HD, SD, HSD. Thus, in the comparison:

$$
(H+H S+H D+H S D)-(O+S+D+S D) \text {, }
$$

involving all pots, each term on the left differs only by the factor $H$ from the corresponding term on the right, and the whole comparison is an unbiased estimate of the effect of the factor $H$.

As a basis for selecting suitable criteria, other things being equal, the higher the ratio (F), the more suitable the criterion. The value of the ratio depends partly on the degree to which the plant responds to treatment in respect of the criterion in question, and partly on the inherent variability of the criterion, which should be, as low as possible. In the present case where criteria differ in the unit of measurement, the coefficient of variability or standard deviation in terms of the mean (V), is the appropriate statistic for assessing comparative variability.

Assuming a significant value for $F$ in any given case, the average effect of each factor and each interaction can be asșessed by taking into account the appropriate standard error. Thus, in the last paragraph but one, the difference between the sum of all the $H$ pots and the sum of all the non- $H$ will be judged significant if it sufficiently exceeds its standard error. The ratio of the difference to its standard error will be 
Student's $t$, the distribution of which is known, and the critical value of which depends merely on the selected level of probability and the number of degrees of freedom used in determining the error variance. Such differences in terms of their standard errors will here be called Normal differences.

Other things being equal, the best criterion will be that which yields the most Normal differences large enough to be judged significant, or alternatively the largest Normal differences.

The results of the pot experiment can now be considered in the light of these three statistical measures, $F, V$, and $t$. and suitable criteria can then be selected for detailed analysis.

Before doing so there remains a problem, however, in that counts of cysts and larvae are not very suitable for analysis as they stand. Owing to the lethal effects of D-D, the analyses contain both high and low counts, and it is found that low counts are associated with low variability, and vice versa. For tests involving $F$ and $t$ it is essential that the mean and variance should be independent, and some kind of transformation is necessary.

Replicate counts of larvae approximate closely to the Poisson distribution, for which the appropriate transformation is to use the square roct of the number counted. If the standard deviation, rather than the variance, is proportional to the mean, a logarithmic transformation is appropriate. Both have been used on the data of this experiment and the logarithmic one is preferred because, although slightly less accurate than the square root transformation, it is far more useful. The criterion "larvae/gm. of soil" is obtained from the product of "cysts/gm." and "larvae/cyst" and, where logarithms are used, is simply got by adding the two values.

\section{Results : Plant Criteria.}

There are five plant criteria, but the first (Height of haulms) was determined on four occasions and the fourth and fifth have two items each (ware-with-seed and total), giving ten items in all. Analysis of variance applied to the results of each of these ten showed a significant value for $F$ in each case, justifying a detailed examination of cach of the seven components of the Treatments variance. These seven conponents are obtained from the various combinations of the eight treatment totals in contrasting groups of four. as illustrated in the "Analysis" section above for the factor $H$. The contrast yields a difference which, divided by its standard error, gives the Normal difference. $(t)$ for the factor in question. Under any given criterion, those Normal 
differences will be judged significant which exceed the tabulated value of $t$ for the selected level of probability.

Table 1 gives, under each of the ten items of the five criteria, (i) the (-values for each of the single factors and interactions, those significant at the $5 \%$ level being in bold type, (ii) the variance ratio, F, and (iii) the coefficient of variability, $V$. It should be pointed out that all the values in this table are pure numbers without dimensions, "and the ten criterial items are comparable in terms of them. The criteria can now be discussed seriatim.

1. Height of Haulms.

At the 29th day after planting, the potato haulms vary cqnsiderably in height, partly because some are by chance late coming through. The coefficient of variability $(V)$ is very high, and the variance ratio $(F)$ is significant only because, even at this early stage, plants in the stenmsterilized soil have grown more rapidly than the others : $S$ is the only factor with a significantly high $t$-value. From the aspects of low $V$ and high $F$, the best day of the four is the 45th, later ones showing greater variability. The Day-45 results also show two single factors and two interactions to be significant, and the t-values are maximal for $S$ (positive) and SD (negative); the only advantage shown by the data for the 59th day is that the $t$-values for $D$ and $H D$ (both positive) are maximal on that day.

The significant interaction HD occurs only with this criterion; it is significant on two of the four days and positive on all four, and is unlikely to be a chance effect. The other three significant components occur under other criteria.

\section{Colour of Haulms.}

It is interesting that this artificially scored criterion with, as expected, a very high variability, has yet provided Normal differences comparable with those for Height on the 59th day and altogether superior to (say) Weight of ware tubers. There is presumably a correlation between depth of green in the coloration of the haulms and their height. The criterion has no obvious advantages beyond ease- of computation, and is in the nature of a curiosity.

3. Dry Weight of Haulms.

This criterion shares with "Weight of all tubers" an outstandingly high variance ratio and a moderate variability, together with closely comparable $t$-values. Of the two it is by far the more troublesome and time-consuming and there would seem to be no good reason for retaining it. 


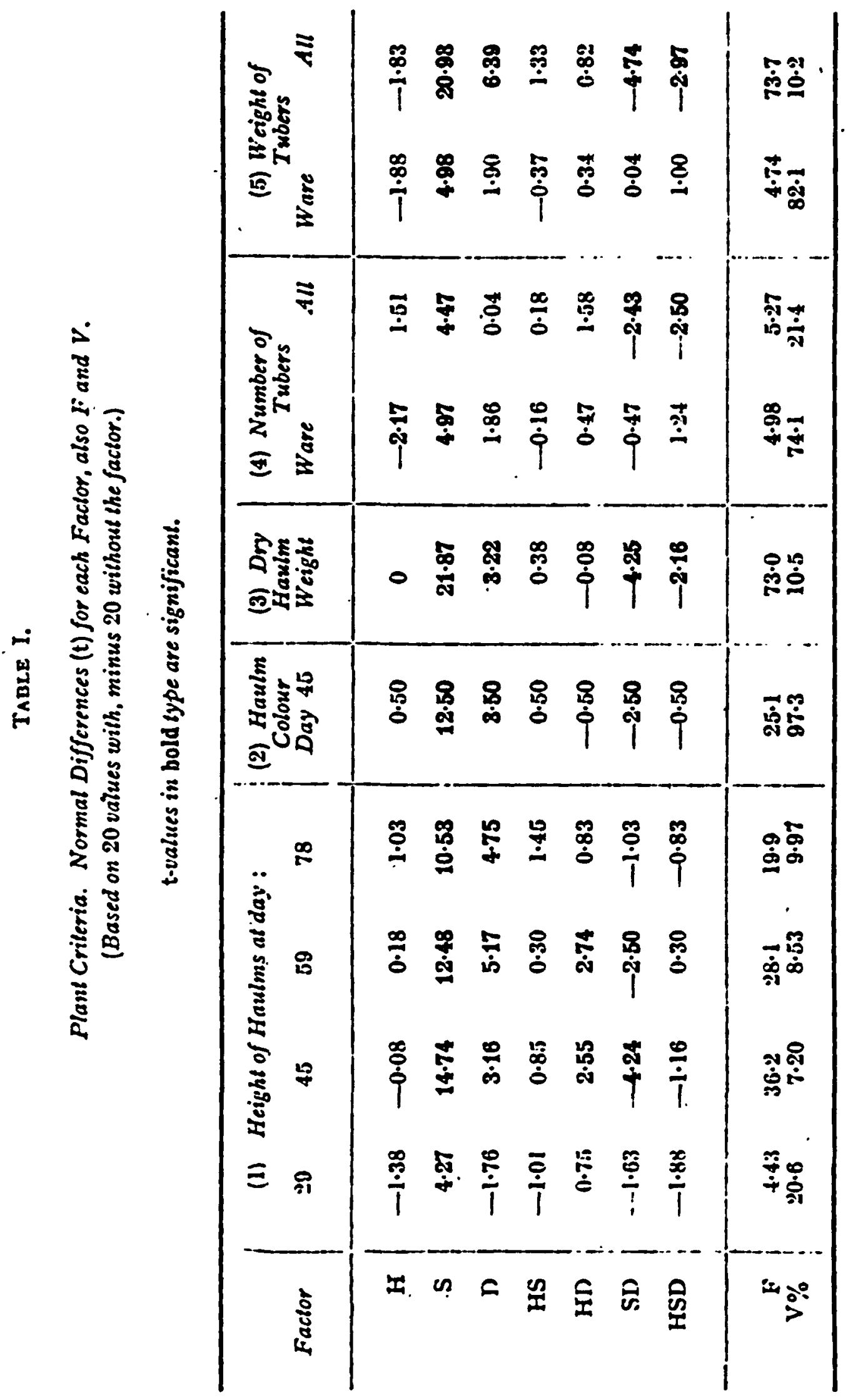




\section{Number of Tubers.}

In this-case the interesting fact is that the number of all potatoes is more sensitive than number of ware-with-seed, having a lower $V$, higher $F$, and three as against two significant $t$-values. The only notable feature of the ware-with-seed analysis is that of all criteria it provides the only significant Normal difference for factor $\mathrm{H}$ : there are fewer ware potatoes in the infected pots.

j. Weight of Tubers.

This criterion resembles the last in that the weight of all potatoes is more sensitive than that of ware-with-seed, only in this case the contrast between the two is far more marked. Weight of all potatoes also is more sensitive than number of all ; it is readily obtained, and in every way recommends itself as a valuable criterion.

\section{General.}

It will be seen that each of the ten criterial items shows a significant positive $S$ effect. Six of them show a significant positive $D$ effect and (not the same six) a negative SD interaction. The heights at 45 and 59 days show a significant positive HD interaction, and the three criteria : dry weight of haulms, number, and weight of all potatoes, a significant negative triple interaction.

It is likely that the experimental results can be fully explored by selecting for detailed consideration the two criteria : height at $\mathbf{4 5}$ days, and weight of all tubers. The only significant effect missed by these two is the negative $H$ effect given by number of ware tubers, and even here the Normal difference for weight of all tubers approaches significance.

\section{Results : Eelworm Criteria.}

The 20 pots containing Heterodera cysts are available for the secondary purpose of investigating the effects of factors $S$ and $D$, and their interaction SD, on the eelworm population as measured by cystand larva-counts. From this aspect, eelworm infection has ceased to be a factor in the experiment $(\mathrm{H})$, and has rather taken the place of the plants as the experimental material under observation.

Table II is similar in construction to Table I and gives $t$-values (Normal differences) and also $\mathrm{F}$ and $\mathrm{V}$ for each of the eelworm criteria. Cyst counts are not suitable for measuring eelworm population, especially in fumigation experiments, because cysts remain in the soil long after their contents have escaped or been killed. Similarly, larvae 
(9)

B. G. PEters

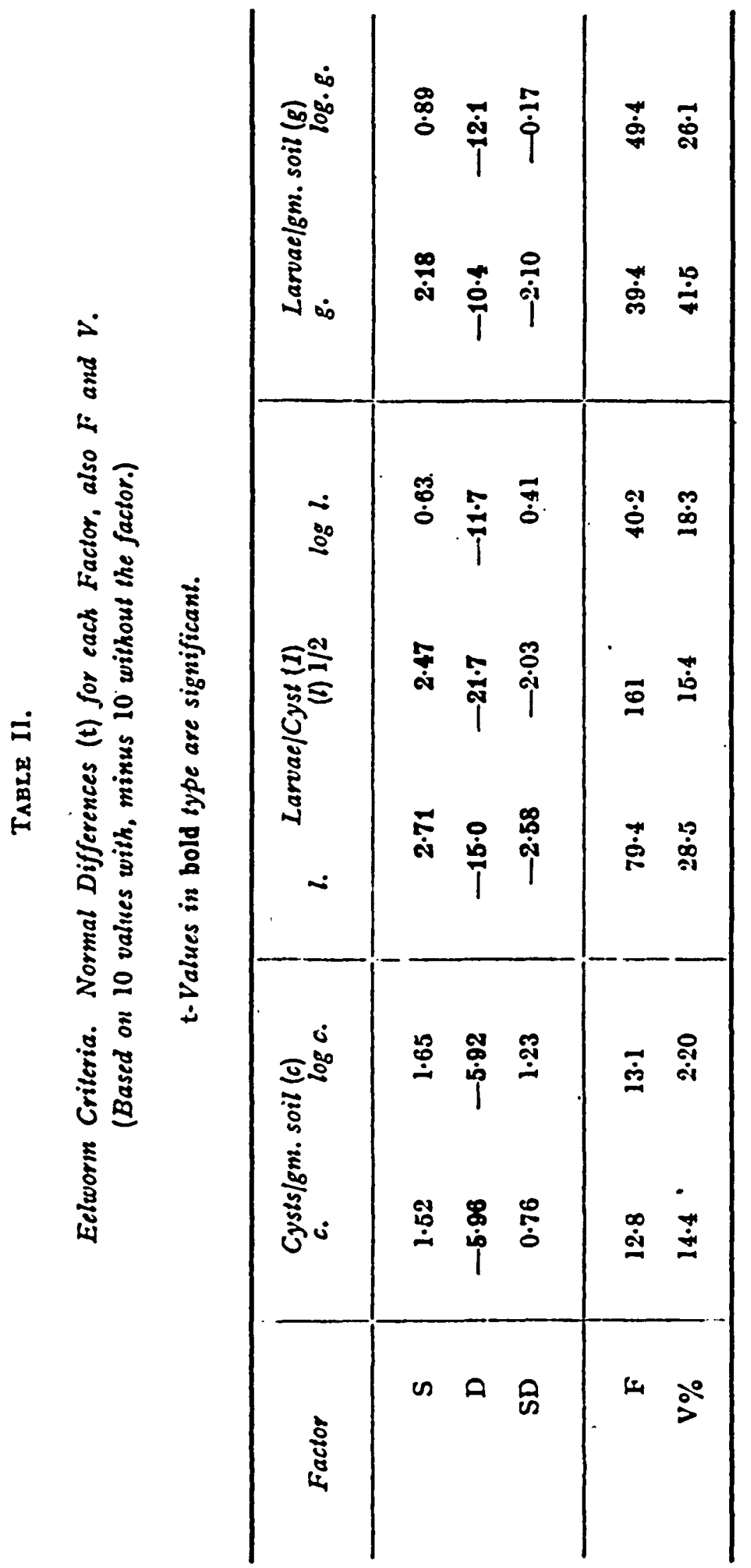


per cyst is a unit which does not directly measure the eelworm population of the soil. The product of these two, larvae per $\mathrm{gm}$. of soil, does give a satisfactory unit, however, provided that one has counted all the living larvae in the sample and no dead ones.

The purpose of Table II is not so much to provide a basis for selecting suitable criteria as to present the statistics of these eelworm distributions and to show the effects of transformations. It will be seen that both cysts/gm. and larvae/cyst (and therefore larvae/gm.) are characterized by high variability before transformation.

TABLE III.

Statistics for Two Distributions of unit:

"Larvae/8 Cysts."

\begin{tabular}{|c|c|c|}
\hline $\begin{array}{lr}\text { Mean Squares : } & \text { (D.F.) } \\
\text { Treatments } & \text { (1) } \\
\text { Pots/Treatment } & \text { (8) } \\
\text { Batches/Pot } & \text { (10) } \\
\text { Aliquots } & \text { (20) }\end{array}$ & $\begin{array}{l}\text { A. D.D Absent. } \\
36906 \\
8246 \\
6427 \\
667 \cdot 15\end{array}$ & 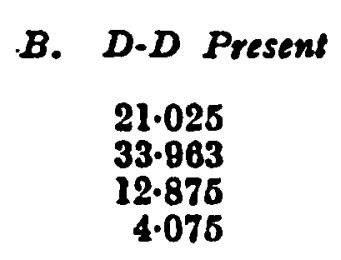 \\
\hline $\begin{array}{c}M e a n(\bar{x}) \\
\text { N. } \\
\text { Variance }\left(s^{2}\right) \\
\text { Standard Dev. (s) } \\
s^{2} / \sqrt[x]{x} \\
s / \bar{x}\end{array}$ & $\begin{array}{c}40 \\
176.3 \\
567.2 \\
23.81 \\
3.22 \\
0.135\end{array}$ & $\begin{array}{l}40 \\
.4 .225 \\
4.075 \\
2.019 \\
0.96 \\
0.478\end{array}$ \\
\hline
\end{tabular}

Table III gives the relevant statistics from a detailed analysis of two sets of counts with the unit: "larvae/8 cysts"; each set comprises 40 counts (duplicate counts from each of two batches of 100 cysts per pot; 10 pots without and 10 with D-D). The data have been separated into these two sets in order to show the relation between the mean and the variability in the extreme cases of high and low counts. It will be seen that the mean varies directly neither with the variance nor with the standard deviation, but somewhere between the two. On this evidence it is not possible to choose between the square-root and the logarithmic transformations, and the latter was in fact chosen because of its usefulness on other grounds. Thus, when working with logarithmic Iransformations (using the terminology of Table II) $\log g$ is the sum of $\log c$ and $\log l$; treatment means when re-transformed become geometric means, and the treatment differences ascribable to factors become ratios. 
Summary and Conclusions.

1. Methods are described whereby a factorial experiment was set up to test the effects on 40 potato plants, growing in $15 \mathrm{~kg}$. pots of soil, of the three factors:-(S) steam sterilization of soil, $(\mathrm{H})$ infection of soil with about three million eggs ard larvae of Heterodera rostochiensis per pot, and (D) injection of soil with $10 \mathrm{ml}$. of D-D per pot.

2. Data from the infected half of the pots were also used to test the effects of factors (S) and (D) on the eelworm population.

3. The data from 10 plant criteria yielded three statistics: the variance ratio (F), the coefficient of variability (V), and the Normal difference $(t)$ corresponding to each factor and interaction. From an examination of these, the following two criteria are selected as, between them, displaying all the significant treatment effects of the experiment :

(a) Height of tallest shoot at 45 days after planting:

(b) Weight of all tubers produced.

4. A consideration of the eelworm counts gives grounds for using a logarithmic transformation of these counts for statistical analysis.

ACKNOWLEDGMENTS.

The writer wishes to thank his colleague, Mr. D. W. Fenwick, for his full assistance in setting up the experiment, and for supervising and taking an active part in the eelworm counts. 